\title{
ON THE REGRESSION OF SENSITIVITY CHARACTERISTICS OF TORQUE TRANSDUCERS
}

\author{
A. Brüge ${ }^{1}$ \\ ${ }^{1}$ Department 1.2, Physikalisch-Technische Bundesanstalt, Braunschweig, Germany, andreas.bruege@ptb.de
}

\begin{abstract}
:
A new method to estimate the influence of regression on the measurement uncertainty for the calibration of torque transducers will be presented in the following. This method is based on Monte Carlo simulations and on sampling calibration results. In some cases, the uncertainty contributions of regression thus obtained are considerably higher than those yielded by estimates according to widely accepted methods. The differences between the methods will be discussed and suggestions for simplified estimates within the scope of the new method will be made.
\end{abstract}

Keywords: Regression; torque; calibration; measurement uncertainty; Monte Carlo simulation

\section{INTRODUCTION}

Calibration results of torque transducers are usually documented in the form of discrete measurement points. These discrete points are then used to calculate a sensitivity characteristic curve by means of a cubic polynomial function, so that users can apply traceability also between those values. In widely accepted methods ([1], [2], [3]), the associated uncertainty contribution is estimated by means of a global characteristic value $u_{f a, S t d}$, assuming a triangular distribution. The conditions justifying such an estimation have not yet been investigated. The introduction of such conditions was mainly motivated by the necessity of keeping the calculation of measurement uncertainties straightforward for users in calibration laboratories. Due to the fact that analytical approaches require considerable effort (see article by D. Röske in these proceedings), systematic investigations have, to date, only been carried out either based on linear regressions or by means of simulation methods ([4], [5], [6]). As a rule, simulations provide results that are specific to a particular application. The special conditions of torque calibration have not yet been sufficiently investigated for this reason. In this paper, the influence of regression on the measurement uncertainty will therefore be examined by means of the Monte Carlo method
(MCM); the results will then be discussed based on real measurement data.

\section{MONTE CARLO SIMULATION}

The dependence of the output signals of strain gauge torque transducers on the load torque is essentially linear. Since the nonlinear contributions are smaller than the linear ones by several orders of magnitude, characteristic curves are mostly represented as linearity deviations over the torque and can be modelled very conveniently by means of a cubic polynomial (Figure 1, green line). The uncertainties associated with each of the data points are used in the MCM to shift the data points accordingly. In the regression, these uncertainties lead to different polynomial functions. In Figure 1, two examples of these polynomials are shown (yellow and orange lines) which are based on the impact of all uncertainty bars in positive and negative directions respectively.

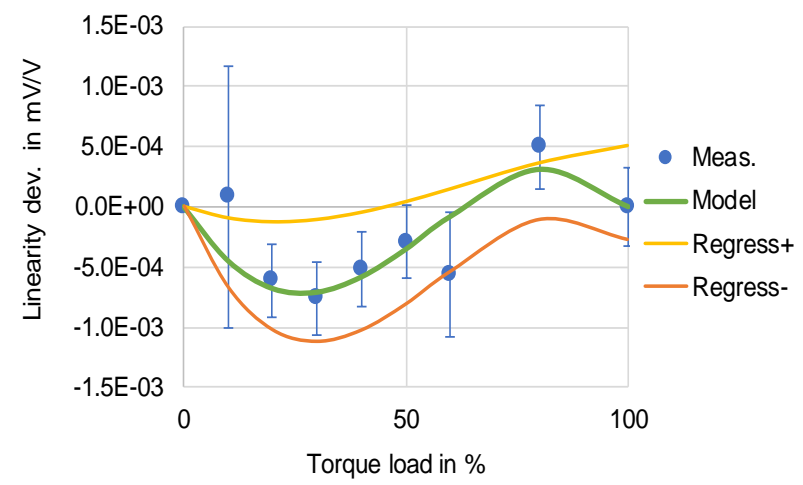

Figure 1: Linearity deviations of a simulated series of measured values (blue dots) over a torque range to which uncertainties (blue bars) are assigned. A cubic regression of the dots provides the green curve. Taking into account the uncertainties, other regression curves are also possible, two of which are shown as examples (yellow and orange).

MCM allows the behaviour of the polynomials to be investigated in detail over a wide range of parameter values. Synthetic measurements as data points are defined by the parameters of linearity deviation $L$, distortion $D$, noise $N$, and the relative 
position of the inflection point $\mathrm{P}_{\mathrm{i}} / \mathrm{NV}$ (Position of inflection point / Nominal Value). The data points are generated with a cubic polynomial in such a way that their maximum deviation from the linear course is given by $L$. With a normal distribution with the standard deviation $D$, the points, which until now have been ideally located on the regression curve, are randomly shifted individually. The simulated measurement curve obtained in this way is now varied randomly within the MCM by shifting the measurement points individually from the distorted centre position generated with $D$, using a normal distribution with the standard deviation $N$. The position of the inflection point $\mathrm{P}_{\mathrm{i}}$ can easily be calculated from the second and third order parameters $c_{2}$ and $c_{3}$ of the cubic polynomial:

$$
\mathrm{P}_{\mathrm{i}}=c_{2} /\left(3 c_{3}\right) \text {. }
$$

For the simulation, the model function is adapted to the conditions of real transducers in certain fields of application by means of typical parameter combinations, as shown in Figure 2. The parameters are obtained by sampling a large number of calibrations $(n \approx 3000)$. From the results obtained by means of MCM, it is possible to gain findings concerning the regression of characteristic curves of transducers.

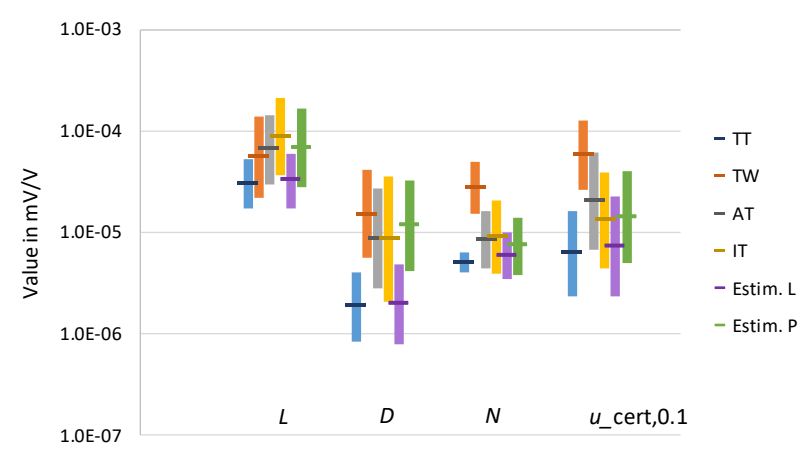

Figure 2: Model parameters in $\mathrm{mV} / \mathrm{V}$ for the simulation of the regression of characteristic curves with a nominal signal of $2 \mathrm{mV} / \mathrm{V}$ in typical fields of application (see the text in Section 4). The values show the expected values (horizontal lines) and the standard deviations (bars) obtained for a sampling of calibrations. Values for the combined standard uncertainty of the calibrations at the $10 \%$ data point $u_{\text {cert, } 0.1}$ are also indicated for comparison purposes.

An analysis of the correlation shows that the measurement uncertainty contributions of interest are mainly influenced by noise $N$. For the standard deviation of the regression deviation $S\left(f_{\mathrm{a}}\right)$, this yields a sensitivity of approximately 4 . The systematic contribution of the regression deviation $u_{f a}$ also depends on the distortion $D$. The linearity and the position of the inflection point do not play a significant role (Figure 3). In the investigated range of values, the influences of $N$ and $D$ are strictly linear. The results of simulations using input parameters with defined values are therefore easily scalable to other input values.

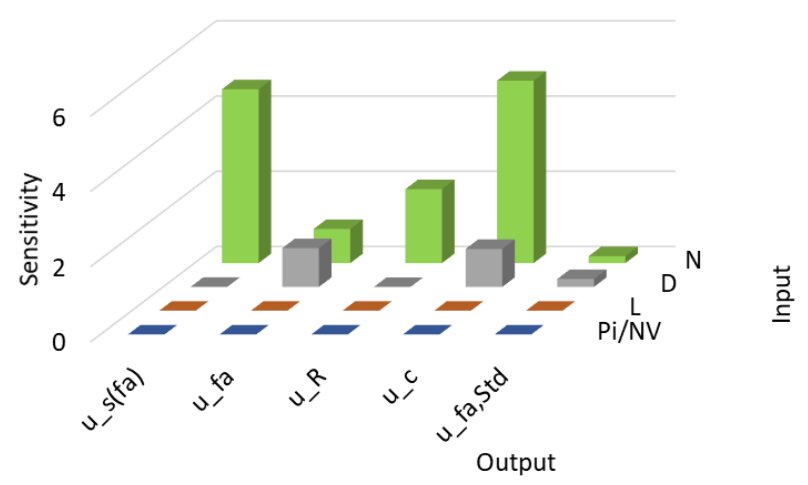

Figure 3: Correlation of measurement uncertainty contributions as output quantities of the MCM simulation of the regression with the model parameters.

The variations of the reference points entering into the simulations $u_{\mathrm{St}}$ are measured in accordance with the noise $N$ which is composed of nonlinearity contributions of the amplifier [7], of its resolution and of the signal disturbance in the measurement chain. Although nonlinearity leads to contributions with beta distribution, the simulations have shown that in realistic scenarios, the contributions of other components with a Gaussian distribution prevail, so that a Gaussian distribution may be assumed. It was possible to confirm this assumption empirically, based on $5 \times 10^{4}$ real data sets.

The uncertainty of the regression function $u_{R}$ must be taken into account when assessing stepped calibration processes between the reference points. This uncertainty is obtained by determining the uncertainty of the polynomial coefficients that define the regression function. For cubic polynomials without an absolute term, simulation between the coefficients of different orders yields normalized covariances with absolute values near 1 :

$\operatorname{cov}_{\text {norm }}\left(X_{i}, X_{j}\right)=\left[\begin{array}{rrr} & -0.9600 & 0.9028 \\ -0.9600 & & -0.9870 \\ 0.9028 & -0.9870 & \end{array}\right]$.

When calculating $u_{\mathrm{R}}$, the covariances may therefore not be neglected.

\section{CONTRIBUTIONS TO THE MEASUREMENT UNCERTAINTY}

\subsection{User's Scenarios}

In current standardization procedures, the influence of the regression enters into the measurement uncertainty in a generalized manner via a plausible estimation. This method does not take the fact into consideration as to whether the user would like to use the regression at all.

This paper therefore suggests considering these parameters separately. When traceability is required at the reference points only (scenario a), the regression and its contribution to the measurement 
uncertainty are dispensable. Only when values between the reference points are also required (scenario b) must this uncertainty contribution be taken into account.

In addition, instead of the generalized estimation of the regression influence, this paper breaks down the contributions into the individual contributions that are made accessible by the MCM simulation: the systematic contribution $u_{f a}$, the random contribution $u_{S f a}$, and the contribution that is attributed to the model $u_{\mathrm{R}}$. Typical curves of these different contributions in the torque range compared to the estimation according to accepted procedures $u_{f \mathrm{a}, \mathrm{Std}}$ have shown that $u_{\mathrm{R}}$ cannot be used as the sole contribution to the uncertainty due to regression (Figure 4). Instead, $u_{\mathrm{S} f \mathrm{a}}$ contains both this contribution and the interaction of the regression with the uncertainty of the reference points, so that these two contributions cannot both enter into the measurement uncertainty budget together.

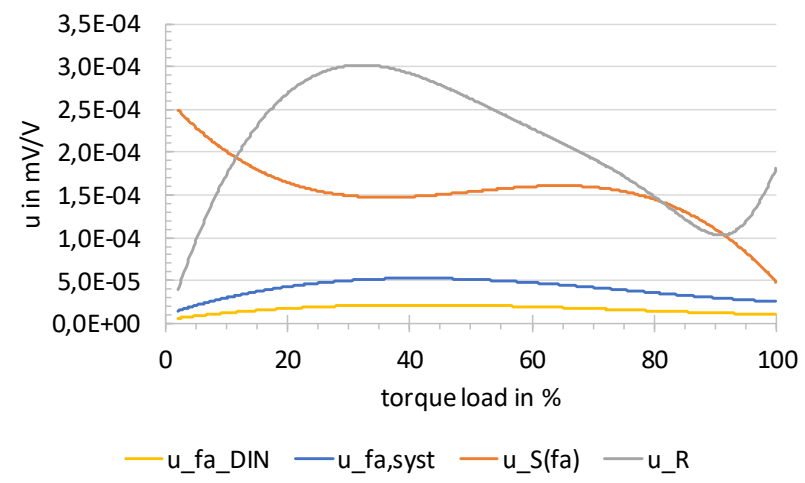

Figure 4: Curves of the measurement uncertainty contributions from the regression for maximum simulation parameters in accordance with the data sampling $\left(N=2 \times 10^{-4} \mathrm{mV} / \mathrm{V}\right)$ compared to the contribution for $f_{\mathrm{a}}$ in accordance with DIN 51309.

The simulations have also shown that the estimates as used in accepted procedures are systematically below the estimations that are determined with the new procedure.

\subsection{Best Estimator}

As long as the standard uncertainty of the systematic fraction $u_{f a}$ is not larger than the uncertainty of the reference points $u_{\mathrm{St}}$, it can be assumed that the reference points, in principle, follow a cubic polynomial curve which therefore represents the "true characteristic curve". The deviations of the reference points from this curve must then be understood as noise, and, due to its averaging action, the regression is the best estimator for the characteristic curve (estimator L). This corresponds the practice of using the average as the estimator of the expected value for quantities with a Gaussian distribution. For the ratio $e=u_{\mathrm{fa}} / u_{\mathrm{St}}>1$, the assumption of a purely cubic characteristic curve is no longer justified, and the reference points represent the best available estimator for the sensitivity of the transducer (estimator P). For practical reasons, the individual values are combined into a geometric sum across all torque steps for this test.

The combined total measurement uncertainty $u_{\mathrm{c}}$ is calculated differently, depending on the user's scenario (a, b) and on the best estimator for the sensitivity (L, P). All contributions to the measurement uncertainty that are not dealt with in this paper are summarized as $u_{\text {rest }}$. Since the sampled calibrations were carried out earlier than 2018 , the contribution of the sawtooth effect $u_{\text {saw }}$ [7] must be added to their uncertainties $u_{c, S t d}^{\prime}$.

The fact that for torque values between the reference points, the curve of $u_{f \mathrm{a}}$ and $u_{\mathrm{S} f \mathrm{a}}$ can only be determined by interpolation must additionally be taken into account for scenario $b$. The interpolation deviations $u_{\mathrm{Int}, \mathrm{S} f \mathrm{a}}$ and $u_{\mathrm{Int}, f \mathrm{a}}$ can be determined at the data points, and an upper estimation with a constant absolute value can be found across the entire torque range (see Section 5.2). From these deviations, the absolute value $u_{\text {Int }}$ is calculated by geometric addition.

$$
\begin{aligned}
& u_{\mathrm{c}, \mathrm{Std}}{ }^{2}=u_{\mathrm{c}, \mathrm{Std}}^{\prime}{ }^{2}+u_{\mathrm{saw}}{ }^{2} \\
& u_{\text {rest }}^{2}=u_{\mathrm{c}, \mathrm{Std}}{ }^{2}-u_{f \mathrm{a}}{ }^{2}-u_{\mathrm{r}}{ }^{2}-u_{\mathrm{A}}{ }^{2} \\
& u_{\mathrm{c}, \mathrm{a}, \mathrm{L}}^{2}=u_{\text {rest }}{ }^{2}-u_{\mathrm{saw}}{ }^{2}+u_{\mathrm{R}}{ }^{2} \\
& u_{\mathrm{c}, \mathrm{b}, \mathrm{L}}{ }^{2}=u_{\text {rest }}{ }^{2}-u_{\mathrm{saw}}{ }^{2}+u_{\mathrm{R}}{ }^{2}+u_{\mathrm{Int}}{ }^{2} \\
& u_{\mathrm{c}, \mathrm{a}, \mathrm{P}}^{2}=u_{\text {rest }}^{2}+u_{\mathrm{r}}^{2}+u_{\mathrm{A}}^{2} \\
& u_{\mathrm{c}, \mathrm{b}, \mathrm{P}}{ }^{2}=u_{\text {rest }}{ }^{2}-u_{\mathrm{saw}}{ }^{2}+u_{f \mathrm{a}}{ }^{2}+u_{\mathrm{Sfa}}{ }^{2} \\
& +u_{\text {Int }}^{2} \\
& =u_{\text {rest }}^{2}-u_{\text {saw }}^{2}+\left(\frac{f_{\mathrm{a}}}{2}\right)^{2}+S\left(f_{\mathrm{a}}\right)^{2} \\
& +u_{\text {Int }}^{2}
\end{aligned}
$$

\section{EMPIRICAL RESULTS}

Real calibration results were analysed correspondingly to the evaluation for the synthetic measurement data. First, the data were classified into categories corresponding to the field of application of the torque transducers and exhibited different sample sizes (TT: 1466 transfer transducers, TW: 100 transfer wrenches, AT: 117 application transducers, IT: 208 industrial transducers). Although this classification is arbitrary and does not provide any clear delimitation, it is nevertheless significantly reflected in the results. In addition, apart from the 
application categories, the data were also arranged according to the best estimator (1550 L, $341 \mathrm{P})$.

When plotting across the interpolation uncertainty, it turned out that the categories TT and TW could mainly be treated with the estimator L (Figure 5). However, there are also many cases where $e>1$. For the categories AT and IT, the distribution is not clear. The correct approach for the sensitivity estimator must therefore be determined individually for each transducer by determining $e$. If $e$ is not known, then only the estimator $\mathrm{P}$ is justified.

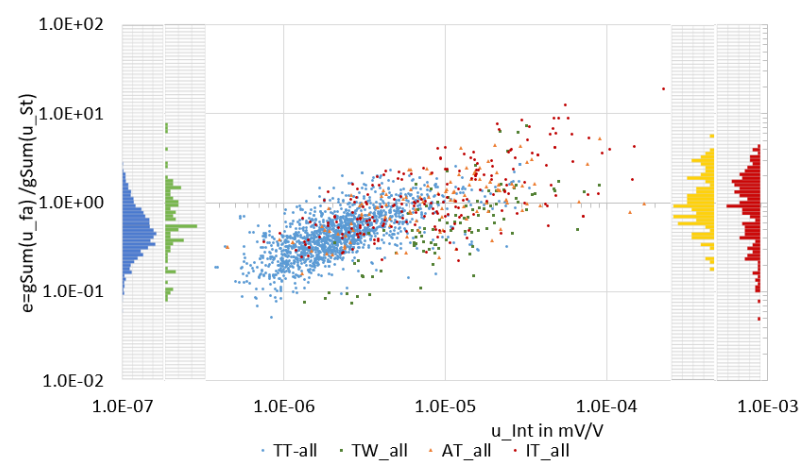

Figure 5: Ratio $e$ of the regression deviation $u_{f a}$ to the uncertainty of the reference points $u_{\text {St }}$ against the interpolation uncertainty $u_{\text {Int }}$. The geometric sums across all reference points were considered. The data are categorized by field of application of the transducers. For each category, the distributions are indicated on either side.

The combined standard measurement uncertainties determined in accordance with equations (5) to (8) by varying the data points by means of MCM and normalized to $2 \mathrm{mV} / \mathrm{V}$ are compared in the following with the corresponding standard measurement uncertainty obtained by means of the accepted procedures:

$$
u_{c, \mathrm{Std}}^{2}=u_{\text {rest }}^{2}+u_{f \mathrm{a}}^{2}+u_{\mathrm{r}}^{2}+u_{\mathrm{A}}^{2} \text {. }
$$

The assessment of the calibration data in scenario a for estimator $\mathrm{L}$ shows that the measurement uncertainty in accordance with the accepted procedures is higher than with the new procedure. Here, the new procedure thus leads to lower measurement uncertainties, in particular for transducers with measurement uncertainties of less than $1 \times 10^{-5} \mathrm{mV} / \mathrm{V}$ (Figure 6, top).

For scenario a and estimator $\mathrm{P}$, the accepted procedure is compatible with the new procedure (Figure 6, centre). In this setting, underestimations remain smaller than a factor of 2 .
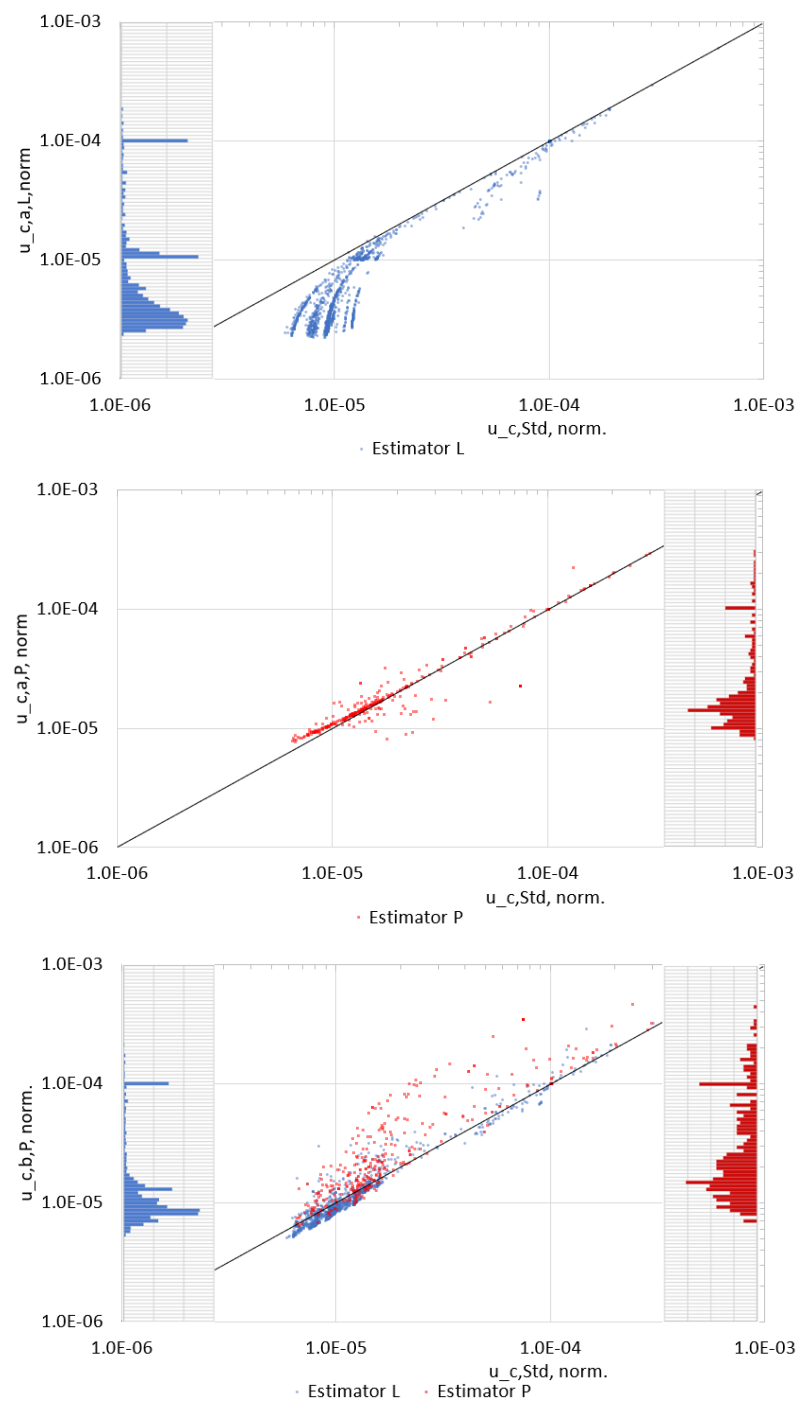

Figure 6: Compared to the combined standard measurement uncertainty normalized to $2 \mathrm{mV} / \mathrm{V}$ from the sampling of real calibration data for the estimator $\mathrm{L}$ and scenario a, the combined standard measurement uncertainty in accordance with accepted procedures is an upper estimate (top).

For the estimator $\mathrm{P}$ and scenario a, the combined standard uncertainty is a suitable estimate (centre), and for the estimator $\mathrm{P}$ and scenario $\mathrm{b}$, it is an underestimate (bottom). The suitable data points that are plotted here for control purposes for the estimator $\mathrm{L}$ are unremarkable.

In the case of scenario $b$ with estimator $P$, underestimations of up to a factor of 4 occur with the accepted procedures. These underestimations are encountered over the whole uncertainty scale and are therefore not correlated with the quality of the transducers. Thus, it is always recommendable to analyse the uncertainty contribution of the regression in more depth if $e>1$ (i.e. in the event of a large regression deviation and small uncertainty of the reference points) and if the calibration result between the reference points is needed. 


\section{SIMPLIFIED ESTIMATION}

Calculating the measurement uncertainty contributions from the use of the regression with MCM requires increased computational effort, which may impede the use of the procedure in a calibration laboratory. It is therefore desirable to find practicable estimation procedures that work without MCM.

\subsection{Estimating $u_{S f a}$ and $u_{R}$ with Boundary Values}

To determine the standard deviations of the residuals $S\left(f_{\mathrm{a}}\right)$ and of the polynomial coefficients of the regression function $S\left(a_{\mathrm{i}}\right)$, using MCM requires numerous variations of the reference points within the limits of their measurement uncertainty and the associated regressions to be calculated. Simplified estimations $S^{\prime}$ of these standard deviations are obtained by calculating only selected variations of the reference points which take the boundary values of the regression functions into account. For this purpose, each of the reference points is varied with the maximum absolute values $N$ of the associated uncertainties and the standard deviation of the residuals and of the polynomial coefficients, respectively, is calculated. To estimate the standard deviation of the residuals, the reference points are varied alternately with $+N$ and $-N$, the regression is performed for each of them, and the standard deviation of these two states is calculated:

$$
S^{\prime}\left(f_{\mathrm{a}}\right)=S\left(f_{\mathrm{a},+-\mathrm{N}}, f_{\mathrm{a},-+\mathrm{N}}\right)
$$

For the simplified estimation of $S^{\prime}\left(a_{\mathrm{i}}\right)$, the reference points are continuously varied with $+N$ and $-N$, respectively, the regression is performed for each of them, and the standard deviations of the resulting polynomial coefficients are calculated. The standard uncertainty for the entire torque range can then be calculated based on these coefficients:

$$
S^{\prime}\left(a_{\mathrm{i}}\right)=S\left(a_{\mathrm{i},+N}, a_{\mathrm{i},-N}\right)
$$

Compared with the corresponding MCM calculations, the simplified calculations generally turn out to be upper estimates in real measurement data (Figure 7). Also in simulations, the simplified calculation leads to combined uncertainties which are slightly higher than combined measurement uncertainties when using the MCM (Figure 8).

\subsection{Constant Estimation}

As shown by the MCM investigations with synthetic signals, the random contributions $S\left(f_{\mathrm{a}}\right)$ and $u_{\mathrm{R}}$ primarily depend on the uncertainty of the reference points $N$ (see Figure 3 ). It therefore suggests itself to estimate the combination of the two contributions $u_{\mathrm{p}, \mathrm{c}}$ using this quantity. Compared to the full MCM calculation, the simulation provides a reliable upper estimation in a contribution $u_{\mathrm{p}, \mathrm{c}} \approx 2 N$ that is constant across the torque range. The constant estimation thus yields a performance similar to that of the estimation of the boundary values. However, its disadvantage is that it leads to a slight overestimation towards the ends of the torque range.

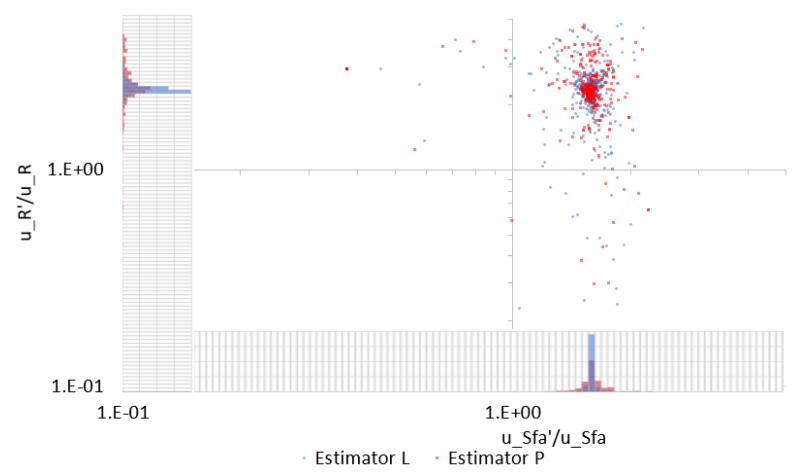

Figure 7: Relationships between simplified calculations of $u_{R}^{\prime}$ and $u_{S f \mathrm{a}}^{\prime}$ and MCM calculations of $u_{R}$ and $u_{\mathrm{S} f \mathrm{a}}$, for real measurement data. Both ratios remain generally above 1, so the simplified calculations are upper estimates.

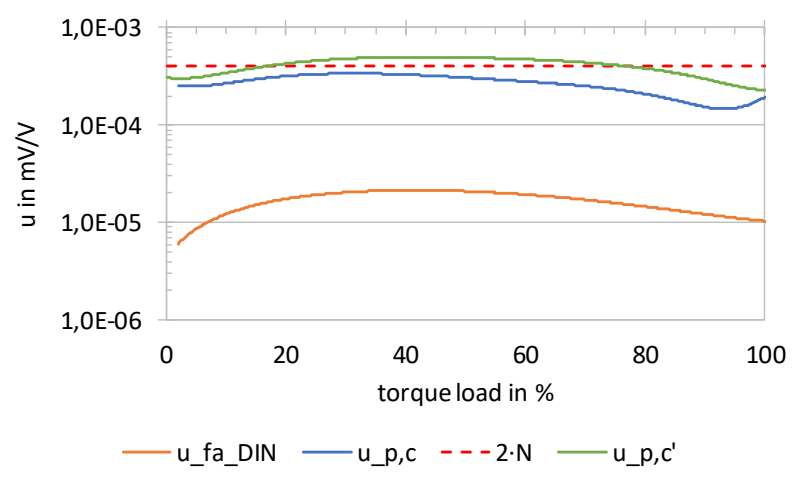

Figure 8: Simulated curves of the combined measurement uncertainties for regression with MCM $\left(u_{\mathrm{p}, \mathrm{c}}\right)$, with boundary value estimation $\left(u_{\mathrm{p}, \mathrm{c}^{\prime}}\right)$, and with the estimation $2 N$ compared to $u_{f_{\mathrm{a}}}$ in accordance with DIN 51309. The simulation parameters correspond to the maxima of the values obtained by sampling real measurements.

\subsection{Interpolation Uncertainties of $\boldsymbol{u}_{f \mathrm{a}}$ and $\boldsymbol{u}_{\mathrm{S} f \mathrm{a}}$}

The contributions to the measurement uncertainty of the residuals $u_{f a}$ and of the variance of the residuals $u_{\mathrm{S} f \mathrm{a}}$ may only be calculated at the reference points. In between the reference points, they have to be interpolated by means of a cubic regression (see Section 3). The resulting uncertainty is yielded from the residuals of these regressions, for which a constant absolute value can be found as an upper estimation by means of its maxima in the torque range.

In the sampling, $u_{\mathrm{S} f \mathrm{a} \text {,Int }}$ appears to be most of the time considerably smaller than $u_{\mathrm{S} f \mathrm{a}}$ (Figure 9, top) and does not represent a dominant contribution in the overall uncertainty budget. In contrast to this, the value of $u_{f a \text {,Int }}$ is similar to that of $u_{f \mathrm{a}}$, which is 
most of the time smaller than $1 \times 10^{-5}$ for calibrations in accordance with estimator $\mathrm{L}$ and thus does not compromise the limits of the overall measurement uncertainty. Contributions for calibrations in accordance with estimator $P$ range up to $1 \times 10^{-4}$ and are therefore not negligible (Figure 9, bottom).
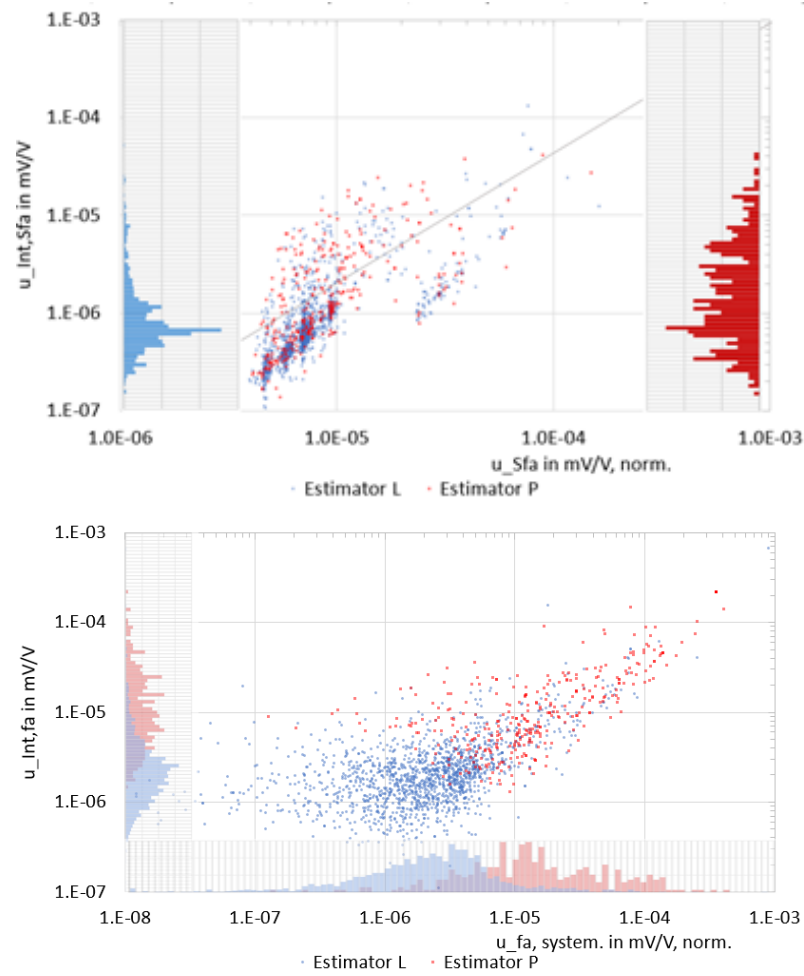

Figure 9: Interpolation uncertainty for $S\left(f_{\mathrm{a}}\right)$ as upper estimation in the entire torque range, calculated as the maximum of the residuals of a cubic fit to the curve of $S\left(f_{\mathrm{a}}\right)$, plotted against the value $S\left(f_{\mathrm{a}}\right)$ at the first load step (top).

Interpolation uncertainty for $f_{\mathrm{a}}$ as the upper estimation in the entire measuring range, calculated as the maximum of the residuals of a cubic fit to the curve of $u_{f a}$, plotted against the value $u_{f \mathrm{a}}$ at the first load step (bottom).

\section{SUMMARY}

Estimating the contribution of the regression to the measurement uncertainty as described in accepted procedures is not always appropriate according to the results of MCM simulations and to the assessment of numerous real calibrations. If the systematic uncertainty of the residuals is larger than the standard uncertainty of the reference points and if the calibration results between the reference points are needed, then the measurement uncertainty obtained by means of accepted procedures may be underestimated by more than a factor of 4 .

A detailed measurement uncertainty analysis can take the requirements of the user of a calibration into account (whether the calibration values at the reference points only or those between the reference points are needed) in different cases. Whether the reference points or the regression must be considered the best estimator for the characteristic curve of the transducer can be calculated in specific approaches.

The new procedure avoids underestimation, but also involves high analytical effort. Simplified procedures, either in the form of the estimation of the boundary values or of a constant upper estimation, can reproduce the results of the suggested procedure with reasonable effort.

\section{REFERENCES}

[1] DIN 51309 - Materials testing machines Calibration of static torque measuring devices, 2005.

[2] "Statische Kalibrierung von anzeigenden Drehmomentschlüsseln" (in German): Guideline DKD-R 3-7, 2018.

Fehler! Linkreferenz ungültig.

[3] "Statische Kalibrierung von Kalibriereinrichtungen für Drehmomentschraubwerkzeuge" (in German): Guideline DKD-R 3-8, 2018.

Fehler! Linkreferenz ungültig.

[4] R. R. Cordero, P. Roth, "On two methods to evaluate the uncertainty of derivatives calculated from polynomials fitted to experimental data", Metrologia, vol. 42, pp. 39-44, 2005.

[5] A. Silva Ribeiro, J. Alves e Sousa, C. Oliveira Costa, M. Pimenta de Castro, "Uncertainty propagation in multi-stage measurements using linear regression analysis and Monte Carlo simulation" in Proc. of the XVIII IMEKO World Congress, Rio de Janeiro, Brazil, 17-22 September 2006.

[6] M. Schalles, M. Hohmann, "Einsatz von MonteCarlo-Methoden zur Bestimmung der Kennlinienunsicherheit" (in German), VDI-Berichte Nr. 2319, pp. 151-162, 2017.

[7] M. F. Beug, H. Moser, A. Kölling, "Bridge Amplifier Linearity Investigation with a Cascaded Inductive Voltage Divider Setup", in Proc. of the XXII IMEKO World Congress, Belfast, United Kingdom, 3-6 September 2018. 